\title{
Original positioning method to determine the clinical and radiographic parameters of the hip joint in patients with cerebral palsy
}

\author{
Summary \\ Relevance: Obtaining true radiographic parameters of the hip joint helps to choose \\ therapeutic tactics for children with cerebral palsy. \\ Goal of the study: Improvement of the diagnostic results in pathology of the hip joint \\ among patients with cerebral palsy by using our original method. \\ Materials and methods: The number of examined patients - 30 persons (60 joints), \\ 15 boys and 15 girls, 26 joints were operated. The age of patients ranged between 3-15 \\ years. All patients underwent a clinical evaluation - Ruwe's femoral torsion, as well as \\ radiography of the hip joints using our original method (utility model patent №137567). \\ Results. The offered method is cheap, simple and accessible to all medical institutions with \\ an X-ray room, for the diagnosis and screening of pathology of the hip joint. \\ Conclusions. The our original method can be used to determine all the main parameters of \\ the hip joint (femoral torsion, neck-shaft angle, Viberg angle, Reimers' index, acetabular \\ angle, Sharp's angle) in patients with cerebral palsy. Making only single radiograph, one \\ obtains true radiographic parameters of both hip joints, which significantly reduces the \\ radiation load upon the patient. Our method can be used in the examination and screening \\ of patients with developmental disorders and other diseases of the hip joint.
}

Keywords: cerebral palsy, hip joint, hip torsion, neck-shaft angle, X-ray parameters
Volume 13 Issue 4 - 202I

\author{
Yatsuliak M, Nemesh M, Martsyniak S, \\ Kabatsiy M, Filipchuk V \\ SI "Institute of Traumatology and Orthopedics of the National \\ Academy of Medical Sciences of Ukraine", Kyiv, Ukraine

\begin{abstract}
Correspondence: Yatsuliak Mykhailo Bogdanovych, Orthopedics of NAMS of Ukraine", 27 Bulvarno-Kudriavska St., Kyiv, 0I06I, Ukraine, Tel +38(097)2655377
\end{abstract} \\ postgraduate student, SI "Institute of Traumatology and \\ Email myhail52368@gmail.com
}

Received: May 26, 2021 | Published: August 16, 2021

\section{Introduction}

In the modern literature, much attention is paid to the treatment of hip joint's pathology among patients with cerebral palsy. Stable, painless, with sufficient range of motion, the hip joint is seen not only as an important tool of ambulation, but also as a necessary factor for a comfortable seating in non-ambulating groups. ${ }^{1}$ Prevention of subluxation, dislocation and contractures in the hip joint among patients with cerebral palsy, who can walk independently or with crutches, and for those who can not ambulate independently should be the goal of modern orthopedics.

Clinical assessment of movement is insufficient to objectively assess the condition of the hip joints in patients with cerebral palsy. Restriction of external rotation in the hip joint cannot be used as a major marker for the clinical evaluation of increased femoral torsion in abovementioned patients. ${ }^{2}$ At the same time, excessive internal rotation of the thigh can be detected in the underdeveloped posterior edge of the acetabular cavity, which is often observed in cerebral palsy. ${ }^{2}$

The method of clinical evaluation of femoral torsion is a simple, easily accessible method and correlates well with intraoperative data. ${ }^{3}$ This technique is used for clinical evaluation of femoral torsion in patients with cerebral palsy. ${ }^{2}$

The correct positioning of a patient with cerebral palsy when performing radiography of the hip joints requires special attention. Existing radiographic positions to determine all hip parameters are recognized, but in most patients with cerebral palsy they cannot be performed due to limited movement and contracture in the joints. Their significant disadvantage is that they are focused upon the diagnosis of instability in the hip joint, and the obtained parameters of the proximal femur are projection ones (the true parameters are found in special tables).

\section{Goal of the study}

To improve the diagnostic results in the hip joint's pathology among patients with cerebral palsy by our originally developed method.

\section{Materials and methods}

Our data are based on the study of clinical cases of 30 patients ( 15 boys and 15 girls), 60 joints ( 26 of the latter were operated), who were treated in the Institute of Traumatology and Orthopedics of the National Academy of Medical Sciences of Ukraine during 20182020 because of the hip joints' disease associated with cerebral palsy. The gender of the patients was not taken into account, as previous studies did not report significant inter-gender differences. ${ }^{4}$ The mean age of patients was $9.15 \pm 0.57$ years ( 3 to 15 years). They had 2-4 levels according to the Gross Motor Function Classification System (GMFCS). The sample in this study included mainly patients with spastic tetraparesis (18 persons), spastic paraparesis (7 persons) and hemiparesis (5 persons).

All patients underwent clinical evaluation of femoral torsion by Ruwe $^{3}$ (Figure 1A-1C), besides we have used our original method (Figure $2 \mathrm{~A} \& 2 \mathrm{~B}) .^{5}$ 


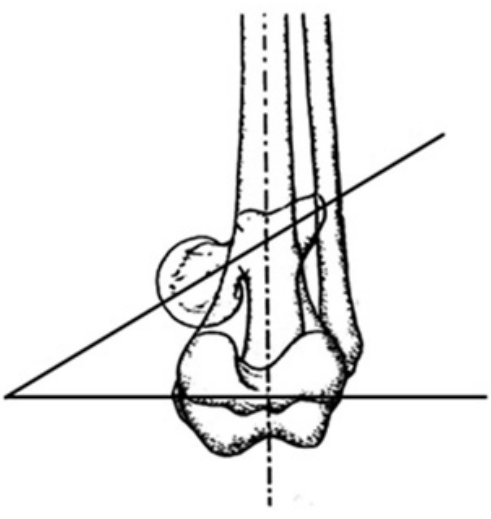

A

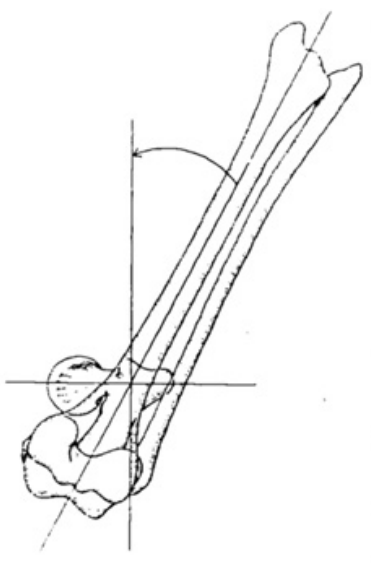

B

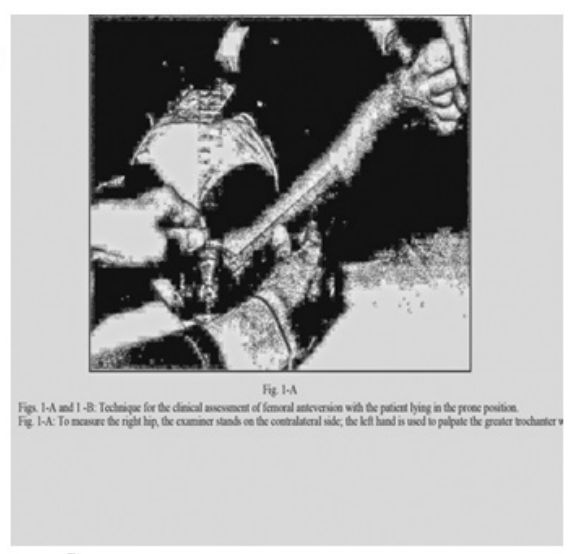

$\mathrm{C}$

Figure I The principle of clinical evaluation of torsion (Figures are taken from Ruwe, 1992). ${ }^{3}$

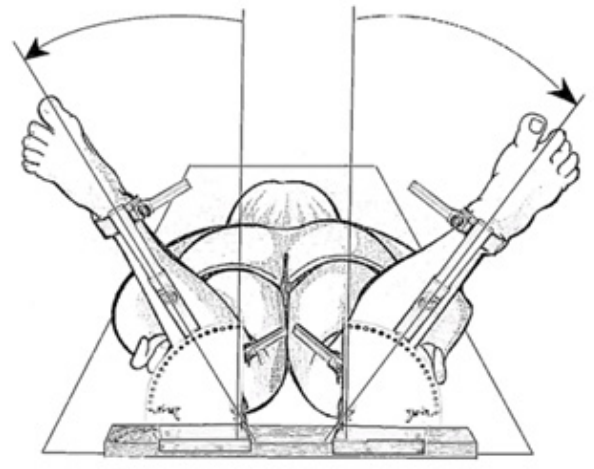

A

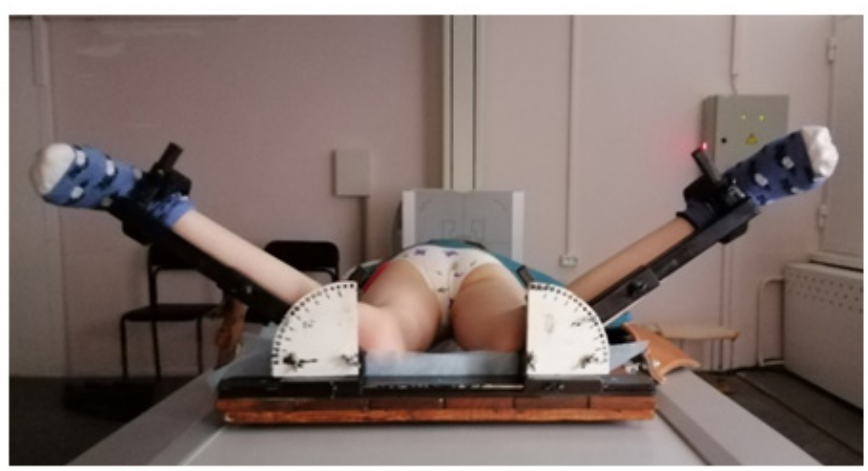

B

Figure $2(\mathbf{A}, \mathbf{B})$ Our original positioning to determine the clinical and radiographic parameters of the hip joint in patients with cerebral palsy.

Formula: the method involves a combination of clinical and radiological methods and their interrelationship, which makes possible to obtain radiographic indicators that exist in different planes when performing only one X-ray imaging of the hip joints (anteriorposterior projection) of the patient in a prone position. In prone position patient's knee joints are flexed at an angle $90^{\circ}$, both thighs are exposed to the internal rotation so that the neck of the femur is positioned parallel to the table (Figure 1B). To do this, the left hand of the doctor was used to palpate the greater trochanter, and with the right hand he performed the internal rotation of the thigh. At a certain point of rotation, the doctor with his left hand noted the maximum palpation of the greater trochanter, which indicates its most lateral position. Fixation of limbs with internal rotation of the thighs and measurement of femoral torsion were performed using developed by authors orthopedic console (patent for the invention №122629 and patent for utility model №140346) (Figure 3). Clinical evaluation of femoral torsion was recorded. The X-ray imaging of hip joints in the described above position was carried out so that we could establish true parameters of a hip joint. ${ }^{5}$

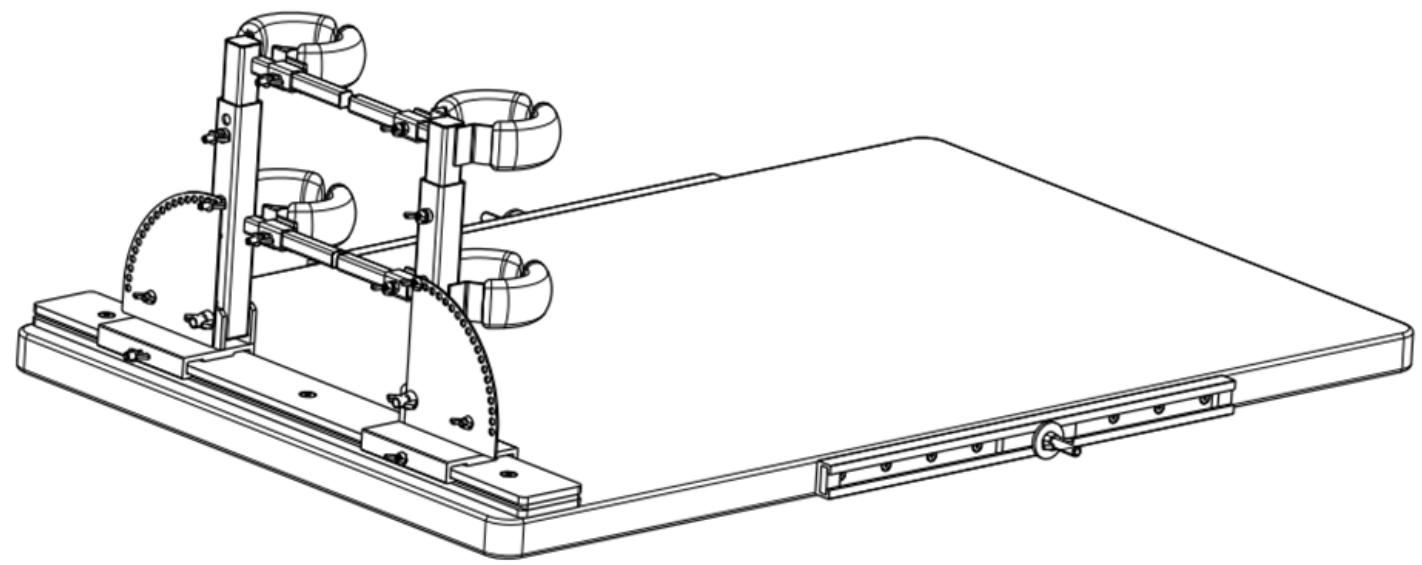

Figure 3 Orthopedic console for radiography of the hip joints (description in the patent application). ${ }^{6,7}$ 


\section{Results and discussion}

The development of a technique for obtaining hip parameters in patients with cerebral palsy was the subject of this study and patients with tetraparesis, paraparesis and hemiparesis, and 2-4 level according to GMFCS were sampled to demonstrate the validity and reliability of the method. Mostly our patients had stable hips and were able to ambulate. Seven thighs had a migration rate $>33 \%$. Some factors that affect the validity and reliability of femoral geometry measurements, such as functional level, degree of femoral instability and bone age, were not analyzed in detail in this article, but such studies will be presented in our future work.

The orthopedic console (Figure 3) is used as follows: the patient is placed in prone position with the lower limbs flexed at an angle 90 degrees at the knee joints on the console and the shins are fixed to the supports. The patient's pelvis is fixed with a belt. We install the brackets with supports so that the femoral abduction was 0 degrees. Alternately and bilaterally one measures the torsion angle of the femur according to the Ruwe's method, sets the femoral torsion angle on the fixing plates and fixes the supports to them with wingnuts at the appropriate angle. The angle reflected on the scale of the fixing plate corresponds to the femoral torsion angle. Hawing performed all steps one can make a radiography of the hip joints. ${ }^{6,7}$

The usage of this console let us fix the patient's limbs at the right angle, which ensures the correct positioning of the patient and guarantees obtaining of objective radiographic parameters. The console gives the possibility to fix the patient's limbs in the specified position, and, accordingly, to obtain radiographic parameters of both hip joints when performing only one X-ray examination. ${ }^{6,7}$

The X-ray examination utilizing the console will allow us to obtain radiographic parameters of the hip joint, which, combined with clinical data, will determine the optimal therapeutic tactics. ${ }^{6,7}$ The described method is simple, accessible and can be used in all medical institutions where an X-ray room is available, for the diagnosis of the hip joint pathology, as well as for screening. When an orthopedic console is absent, the researcher can determine the femoral torsion angle with an ischiometer and perform an X-ray of the hip joints in case of the femoral internal rotation at a certain torsion angle while holding the patient's limbs independently.

The criteria for assessing the positioning correctness using our original method are the same ones as in the standard anterior-posterior positioning. If the femoral torsion is reliably determined - the radiographs clearly display the growth zone of the greater trochanter, and the shadow of the latter is not superimposed upon the shadow of the femoral neck. Properly determined femoral torsion provides obtaining of the true parameters of hip joint.

On each radiograph we recommend to mark the angle of femoral internal rotation and right and left sides (Figure 4) to avoid diagnostic and subsequent therapeutic errors.

A discussion may be elicited referring obtaining the true parameters of neck-shaft angle and torsion when performing the radiograph using our original method in case of axial deformities of the limbs when the patient lies in prone position, thighs are rotated internally at a torsion angle measured by Ruwe (between the axis of the shin and perpendicular in the most lateral position of the greater trochanter). Axial deformities of the extremities (varus, valgus) are not typical for cerebral palsy, but if they are present, the error in the angle of axial deformation should be taken into account (if the knee valgus is $10^{\circ}$, the torsion angle measured by Ruwe's method will be by $10^{\circ}$ less, in knee varus it is higher). ${ }^{2,3}$

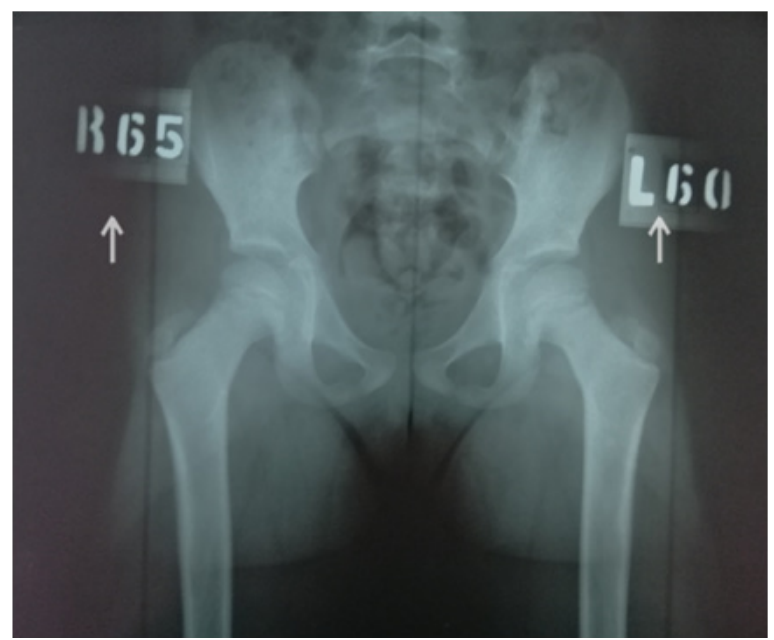

Figure 4 Radiograph of the hip joints using our original method (the arrows indicate the angle of femoral internal rotation on the right and left, respectively, which corresponds to the clinically defined torsion of the femurs according to Ruwe).

The examination of obese patients and patients less than 3 years old, including palpation of the greater trochanter, caused some difficulty. ${ }^{2,3}$ This study did not include adult patients with cerebral palsy, in this group with age the range of movements in the hip joints decreases due to the formation of neurogenic contracture and then we observed a situation when the magnitude of femoral torsion was much greater than internal rotation. These patients are often admitted into our clinic for therapeutic reasons. In this case, we recommended radiography in the position of maximal femoral internal rotation. Then radiographic parameters of the hip joint are as close as possible to the true ones.

There may be a discussion regarding the patients with severe movement limitations and contractures. Positioning according to our original method is preceded by clinical evaluation of femoral torsion by Ruwe, if a severe somatic condition does not allow the patient to be positioned for this study, we are forced to abandon the functional positioning according to our original method, as in such a complex category of patients accurate radiographic parameters of the hip joint are not primary ones, and reconstructive surgery is replaced by palliative one.

Our method of determining the clinical and radiographic parameters of the hip joint in patients with cerebral palsy can replace CT scan, although some authors have questioned the usage of computed tomography to determine the torsion of the femur. ${ }^{8}$ The results of measuring the torsion of the femur by CT can have significant differences $(\mathrm{P}<0,001)$ depending on the measurement technique. ${ }^{9}$ Hip flexion is a major source of inaccuracy on CT scans, suggesting that inaccurate measurements are likely in patients with neuromuscular disorders, such as cerebral palsy, who have limitation of movements and contractures. ${ }^{10}$

\section{Conclusions}

- Our method is simple and reliable for determining not only the instability of the hip joint, but also the precise parameters (femoral torsion, neck-shaft angle, Viberg angle, Reimers' index, acetabular angle, angle of acetabular inclination) in patients with cerebral palsy. 
- The obtained radiographic parameters of hip joints bilaterally, when performing only one X-ray, also significantly reduce the radiation load upon the patient, as patients with cerebral palsy are subject to regular X-ray examination during the entire period of joint formation.

- The method can also be used in the examination and screening of patients with developmental disorders and other diseases of the hip joint.

\section{Acknowledgments}

None.

\section{Conflicts of interest}

The authors declare the absence of a conflict of interest during the preparation of this article.

\section{Gratitude}

The authors express their gratitude for the fruitful cooperation to a friend, teacher and colleague V. Hoshko (August 4, 1949 - September $28,2020)$.

\section{References}

1. Bleck EE. Orthopedic management cerebral palsy. Oxford, Philadelrhia. Mac Keith Press; 1987. 405p.

2. Chung CY, Lee KM, Park MS, et al. Validity and reliability of measuring femoral anteversion and neck-shaft angle in patients with cerebral palsy. $J$ Bone Joint Surg Am. 2010;92(5):1195-205.
3. Ruwe PA, Gage JR, Ozonoff MB, et al. Clinical determination of femoral anteversion. A comparison with established techniques. J Bone Joint Surg Am. 1992;74(6):820-830.

4. Upadhyay SS, Burwell RG, Moulton A, et al. Femoral anteversion in healthy children. Application of a new method using ultrasound. J Anat. 1990;169:49-61.

5. Hoshko VIu, Naumenko NO, Cheverda AI, et al, inventors; SI "Institute of Traumatology and Orthopedics of NAMS of Ukraine", assignee. The method of determining the clinical and radiographic parameters of the hip joint in patients with pathology of the hip joint. Ukrainian patent №137567. 2019. [in Ukrainian].

6. Hoshko VIu, Naumenko NO, Yatsuliak MB, inventors SI "Institute of Traumatology and Orthopedics of NAMS of Ukraine", HoshkoVIu, Yatsuliak MB, assignees. Orthopedic attachment for laying the patient during radiography of the hip joints. Ukrainian patent №122629. 2020. [in Ukrainian].

7. Hoshko VIu, Naumenko NO, Yatsuliak MB, inventors; SI "Institute of Traumatology and Orthopedics of NAMS of Ukraine", HoshkoVIu, Yatsuliak MB, assignees. Orthopedic attachment for laying the patient during radiography of the hip joints. Ukrainian patent №140346. 2020. [in Ukrainian].

8. Morvan G, Guerini H, Carré G, et al. Femoral Torsion: Impact of Femur Position on CT and Stereoradiography Measurements. AJR Am J Roentgenol. 2017;209(2):W93-W99.

9. Kaiser P, Attal R, Kammerer M, et al. Significant differences in femoral torsion values depending on the CT measurement technique. Arch Orthop Trauma Surg. 2016;136(9):1259-1264.

10. Jarrett DY, Oliveira AM, Zou KH, et al. Axial oblique CT to assess femoral anteversion. AJR Am J Roentgenol. 2010;194(5):1230-1233. 